\title{
Supervisory Control and Data Acquisition system applied to a researching purpose microgrid based on Renewable Energy
}

\author{
Carlos Vargas-Salgado ${ }^{a}$, Jesús Águila-León ${ }^{\text {b,c }}$, Cristian D. Chiñas-Palacios ${ }^{\text {b,c }}$, David \\ Alfonso-Solar ${ }^{\text {d }}$ \\ ${ }^{a}$ Departamento de Ingeniería Eléctrica, Universitat Politècnica de València, Valencia, España, \\ carvarsa@upvnet.upv.es, ${ }^{\mathrm{b}}$ Instituto Universitario de Ingeniería Energética, Universitat Politècnica de \\ València, España, carvarsa@upvnet.upv.es, jeagleo@upvnet.upv.es, cricripa@upvnet.upv.es, \\ ${ }^{\mathrm{c}}$ Departamento de Estudios del Agua y la Energía, Universidad de Guadalajara, México, \\ jesus.aguila@academicos.udg.mx, daniel.chinas@academicos.udg.mx, $\quad{ }^{\mathrm{d}}$ Departamento de \\ Termodinámica Aplicada, Universitat Politècnica de València. Spain, daalso@iiie.upv.es
}

\begin{abstract}
Control and data acquisition systems are required in researching facilities to analyze the behaviour of any process. In this paper, the results of the design and implementation of an automation and control system applied to a microgrid based on renewables energy are shown. The microgrid is located in the Laboratory for Distributed energy resources - LabDER at the Institute for Energy Engineering at UPV. The brain of the system is a PLC, programmed to carry out several tasks to guarantee the correct operation of the system. The measuring devices used are power meters, anemometer, temperature sensors and a solar cell to measure irradiance. The communication protocol used is Modbus TCP IP, Modbus RS-485 and Xanbus. All the information is centralized using a SCADA as an HMI. As a result, a robust control, and data acquisition system, able to manage a microgrid for researching purpose was obtained.
\end{abstract}

Keywords: SCADA, Microgrid, renewable energy, automation and control, data acquisition.

\section{Introduction}

Facing the increase of energy demand of our human society the emerging technologies have provided a new framework along with distributed power generation to increase efficient use of energy (Hirsch, Parag, \& Guerrero, 2018). Renewable energy systems see a pleasant future for society and the environment in the change of power in the centralized generation 
scheme to a distributed power generation in the form of microgrids systems (Zhaoxia, Zhijun, Guerrero, \& Hongwei, 2017). A microgrid is a power generation system consisting of two or more generation sources working together.

Microgrid distributed generation is usually based on renewable energy sources, so energy production is limited by changing environmental conditions, especially for remote areas (Akinyele, Belikov, \& Levron, 2018). Under this context, one way to increase the efficiency of energy production in microgrids is through a Supervisory Control and Data Acquisition (SCADA) system to ensure power supply, quality, and control for distributed generation systems (Iqbal \& Iqbal, 2019). SCADA systems were developed back in 1960, when the solid-state electronics development and first telephonic telemetry networks allowed a broader automated control and monitoring process in the industry, therefore, SCADA systems evolution had been subject to technology and market evolution (Ujvarosi, 2016). The SCADA systems are responsible for collecting sensor data, managing execution tasks, and providing information to the user; these systems are used mainly for industrial applications (Rezai, Keshavarzi, \& Moravej, 2017). Moreover, the renewable energy sources penetration for distributed generation leads to SCADA systems development and implementation for small to medium size applications. Researchers among the world had been using different SCADA systems software and topologies since there are several commercial options to choose. It has been proved that when using a SCADA system, the general efficiency of a microgrid can be increased up to $75 \%$, uniquely when the system is accurately programmed and deployed (Romero, 2018). To achieve better efficiency of the microgrid system is needed to establish control, and therefore, communications are a central part of the process. In industry there are a wide variety of communication protocols, many of them specialized for specific applications, however, in the field of energy control and monitoring there is not any dedicated communication protocol (Bani-Ahmed, Weber, Nasiri, \& Hosseini, 2014). Authors have been using industry-standard protocols for microgrids. As revealed for literature review, it has been found that PROFIBUS (Romero, 2018), CAN (Chen \& Pei, 2013) and Modbus (Arciniegas, Imbajoa, \& Revelo, 2017) protocols are often used, being the selection of the protocol prosecuted by the existing or available hardware, costs and closed brandmark firmware, being the election of the controller device also limited by the same factors. Among the most common controller devices, it is found that PLC is up today the most secure and robust option since these controllers have been in develop for more than forty years (Chowdhury, Chowdhury, \& Crossley, 2009).

In this paper, it is presented the design, architecture, and deployment of a SCADA system for an experimental microgrid for future research and academic purposes. The device controller is an OMRON CJ2M CPU31 PLC, and the Human Machine Interface (HMI) is computer-based. 


\section{SCADA system design and deployment}

\subsection{Microgrid characteristics}

The presented SCADA system was implemented in the Laboratory of Renewable Energies (LabDER) - Universitat Politècnica de València. As shown in Fig. 1, the microgrid in which the web SCADA system was deployed consists of an array of solar photovoltaic panels, a wind turbine, a syngas electric generator, a bank of batteries and a diesel generator as back up.

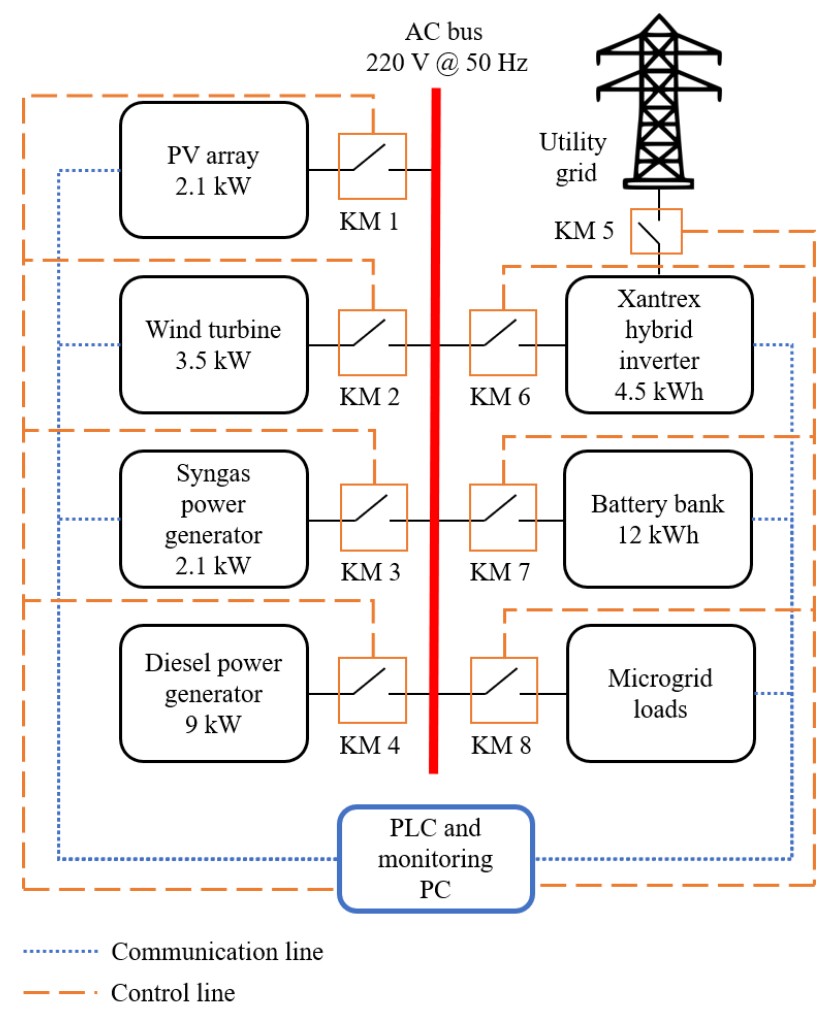

Fig. 1 LabDER microgrid for the presented SCADA system implementation.

The LabDER microgrid can operate in islanded or grid-tied mode. When working in offgrid mode, the microgrid can supply power from the available renewable energy sources or the backup energy storage devices for the inside laboratory energetic needs, computers, ventilators, and air conditioning. Table 1 depicts detailed information about the LabDER microgrid main components. 
Supervisory Control and Data Acquisition system applied to a researching purpose microgrid based on Renewable Energy

Table 1 LabDER microgrid components.

\begin{tabular}{|c|c|}
\hline Description & Features \\
\hline Photovoltaic array & $2.1 \mathrm{~kW}$ \\
\hline Wind power turbine & $3.5 \mathrm{~kW} @$ wind speed of $12 \mathrm{~m} / \mathrm{s}$ \\
\hline Syngas power generator & $10 \mathrm{~kW} @ 30 \mathrm{Nm} 3 / \mathrm{h}$ \\
\hline Battery bank & $12 \mathrm{kWh}, 12 \mathrm{~V} 12 \mathrm{~V} @ 250 \mathrm{Ah}$ \\
\hline Diesel backup generator & 9 kW, 230VAC@50 Hz \\
\hline Xantrex hybrid inverter & $4.5 \mathrm{~kW}, 230 \mathrm{VAC} @ 50 \mathrm{~Hz}$ \\
\hline
\end{tabular}

\subsection{Design and deployment}

The LabDER SCADA system design can be divided into three main layers: the physical layer, the network layer, and the control and supervisory layer. Figure 2 represents the details of every implemented layer in communications.

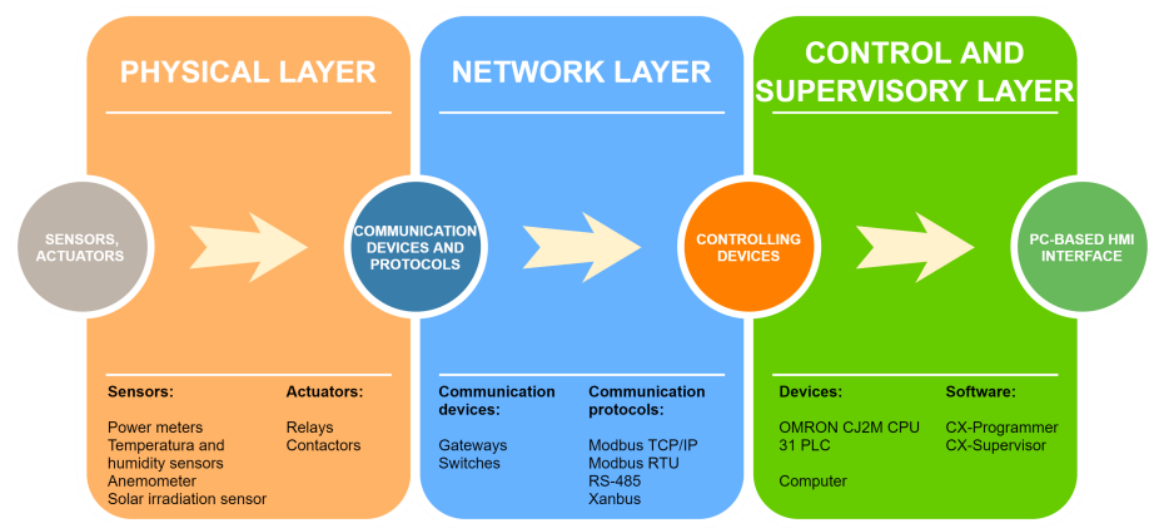

Fig. 2 SCADA LabDER microgrid layers.

Communications are crucial for SCADA systems, since trough them system parameters and conditions are measured, and consequently, controlling actions can be achieved to maintain the microgrid operating under stability conditions. Communications must be stable and secured. The LabDER microgrid was designed for Modbus TCP/IP using gateways for Modbus RTU, RS-485 and Xanbus communications, in such a manner that the RJ-45 infrastructure can be used both for internet communications and for Modbus communications standardized inside de the microgrid. 
For the SCADA system control, the OMRON CJ2M CPU31 PLC was connected to perform actions over the system, reading sensors and linked to the monitoring PC for data storage and supervising. The PLC was programmed using CX-Programmer software, and the SCADA user interfaces using CX-Supervisor software. The PC-based SCADA user interface communicates with the CJ2M to read and write to the PLC registers. Besides, the SCADA can store microgrid measurements in its local database.

\section{Results}

After the design and implementation of the SCADA system, a system was obtained through which the user can review the history of measurements and events performed in the microgrid through the local database implemented in the SCADA system, besides, a friendly interface was obtained for the monitoring, control and monitoring of the microgrid.

\subsection{SCADA system local DB}

A local DB for the LabDER SCADA system was developed. The local DB can store data from every power meter, the anemometer, the environmental temperature and relative humidity as well as microgrid events and alarms. Data is stored locally in different database tables and registers by using automated read and write scripts executed by the CXSupervisor SCADA system runtime interface. Figure 3 (a) shows the DB tables created, and Figure 3 (b) the automated read and write script programmed in CX-Supervisor.

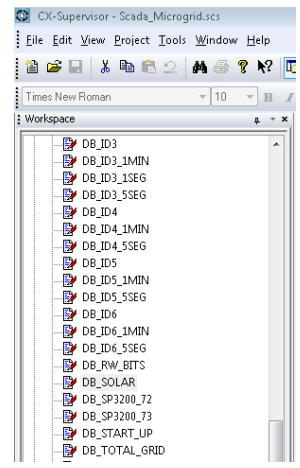

(a)

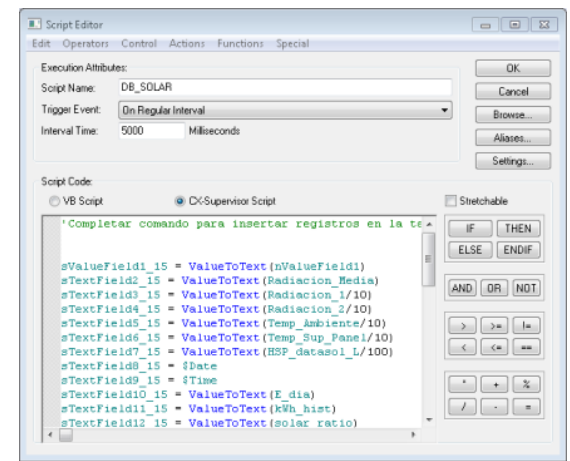

(b)

Fig. 3 (a) local DB tables and (b) automated read and write registers script.

\subsection{SCADA}

User can interact with the SCADA system through a PC-based graphical user interface programmed in CX-Supervisor software. The user interface is a runtime script that must be 
open as long as the microgrid operates in order to keep upper control and monitoring of the microgrid, lower control is programmed in the PLC, so if the SCADA interface is closed the microgrid is controlled under safe conditions by the PLC using pre-programmed operation and alarms rules. The main page of the graphical user interface is showed in Figure 4(a); in this section, the user can have overall monitoring of all the microgrid subsystems. User can navigate in the interface to obtain additional information and details from different devices and subsystems of the microgrid as can be observed in Figure 4 (b) where detailed information about the photovoltaic array is displayed.

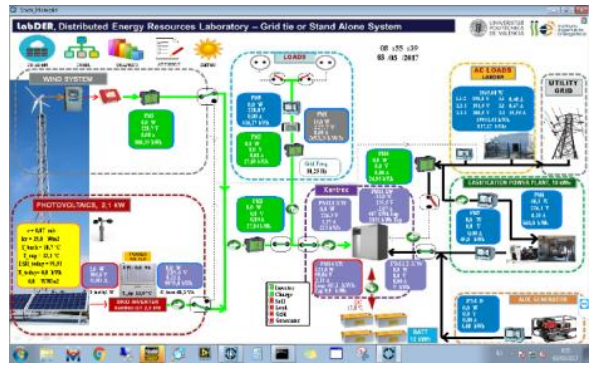

(a)

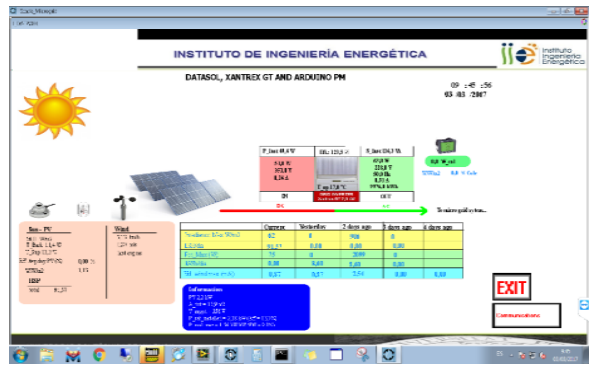

(b)

Fig. 4 (a) SCADA main page and (b) detailed view of the displayed information of the photovoltaic array.

\section{Conclusions}

It is feasible a PLC architecture for monitoring, data collecting and controlling the behaviour and operation of the microgrid since PLC are robust automation devices. Tests have demonstrated excellent performance of the SCADA system, displaying, storing, and analyzing real-time data within the CX-Supervisor software. Moreover, the Modbus TCP/IP protocol exhibited a reliable, fast, stable and robust behaviour in the communication architecture of the LabDER-UPV to the devices connected inside the network, achieving speeds of up to $100 \mathrm{~ms}$, and transfer rates of up to $0.4 \mathrm{Mb} / \mathrm{sec}$.

Furthermore, having am open standard communications protocol such as Modbus TCP/IP helps a subsequent modular growth of the microgrid network, facilitating the addition of new devices. As future work, an in-depth evaluation analysis to the DNP3 protocol compared to the Modbus TCP IP as an alternative to improve data transmission type handling in communications within the microgrid network, as well as the design of an energy management system for the microgrid, would allow the system to self-adjust according to energy demand and the availability of its resources, thus obtaining the maximum possible energy usage. 


\section{Acknowledgments}

The authors gratefully acknowledge to the Universitat Politècnica de València and the Instituto Universitario de Ingeniería Energética for their support to accomplish this work.

\section{References}

Akinyele, D., Belikov, J., \& Levron, Y. (2018). Challenges of Microgrids in Remote Communities: A STEEP Model Application. Energies, 11(2), 432. https://doi.org/10.3390/en1 1020432

Arciniegas, A. F., Imbajoa, D. E., \& Revelo, J. (2017). Diseño e implementación de un Sistema de Medición Inteligente para AMI de la microrred de la Universidad de Nariño (Design and implementation of a Smart Measurement System for AMI in the microgrid of the University of Nariño). Enfoque UTE, 1, 300-314. Retrieved from http://ingenieria.ute.edu.ec/enfoqueute/

Bani-Ahmed, A., Weber, L., Nasiri, A., \& Hosseini, H. (2014). Microgrid communications: State of the art and future trends. 3rd International Conference on Renewable Energy Research and Applications, ICRERA 2014, 780-785. https://doi.org/10.1109/ICRERA.2014.7016491

Chen, Y., \& Pei, W. (2013). Design and implementation of SCADA system for micro-grid. Information Technology Journal, 12(24), 8049-8057. https://doi.org/10.3923/itj.2013.8049.8057

Chowdhury, S., Chowdhury, S. P., \& Crossley, P. (2009). Microgrids and active distribution networks. Microgrids and Active Distribution Networks. IET RENEWABLE ENERGY SERIES. https://doi.org/10.1049/pbrn006e

Hirsch, A., Parag, Y., \& Guerrero, J. (2018, July 1). Microgrids: A review of technologies, key drivers, and outstanding issues. Renewable and Sustainable Energy Reviews. Elsevier Ltd. https://doi.org/10.1016/j.rser.2018.03.040

Iqbal, A., \& Iqbal, M. T. (2019). Low-cost and secure communication system for SCADA system of remote microgrids. Journal of Electrical and Computer Engineering, 2019, 1-13. https://doi.org/10.1155/2019/1986325

Rezai, A., Keshavarzi, P., \& Moravej, Z. (2017, February 1). Key management issue in SCADA networks: A review. Engineering Science and Technology, an International Journal. Elsevier B.V. https://doi.org/10.1016/j.jestch.2016.08.011

Romero, J. (2018). Implementación de un sistema SCADA para el control de una micro red inteligente en zona rural, 1-12. Retrieved from http://repository.udistrital.edu.co/bitstream/11349/13619/1/RomeroPovedaJuanSebastian2018.pdf

Ujvarosi, A. (2016). EVOLUTION OF SCADA SYSTEMS. Bulletin of the Transilvania University of Braşov • (Vol. 9).

Zhaoxia, X., Zhijun, G., Guerrero, J. M., \& Hongwei, F. (2017). SCADA system for islanded DC microgrids. Proceedings IECON 2017 - 43rd Annual Conference of the IEEE Industrial Electronics Society, 2017-Janua, 2669-2674. https://doi.org/10.1109/IECON.2017.8216449 